\title{
Focus Awards 2001
}

\author{
Liane Voisey
}

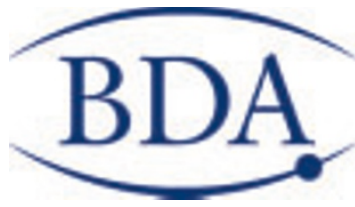

\author{
In the fourth of a series of five articles in which we look at each of the \\ practice finalists from the Focus Awards 2001, we visit Strelley Health \\ Centre in Nottingham, one of the CDS clinics in the region.
}

\begin{abstract}
The Focus Awards are a joint initiative sponsored by the Department of Health and the BDA which recognise and reward patientfocused innovations that have taken place in the last two years. Any single or group of providers of NHS dental care within GDS, CDS or PDS can be nominated. The deadline for applications is 30 June 2002. To apply for this year's Focus Awards or request further information please call the BDA on 020 75634563.
\end{abstract} chased to make the patient experience more comfortable and amenable. Colour schemes were also established to ensure pleasant surroundings and a more relaxed patient atmosphere.

Even in the reception area, the staff at Strelley Health Centre have endeavoured to raise positive awareness of oral health,

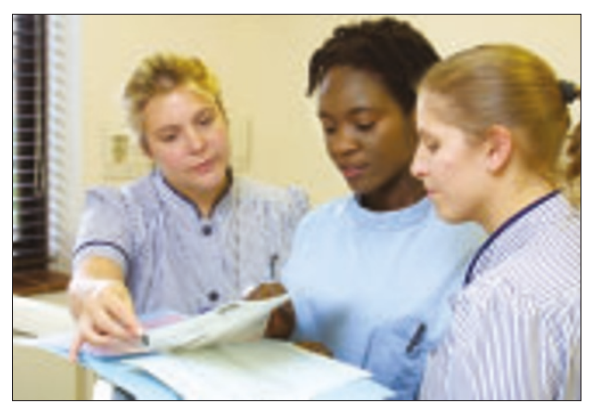

Fig. 2. There is a team-oriented structure at Strelley Health Centre.

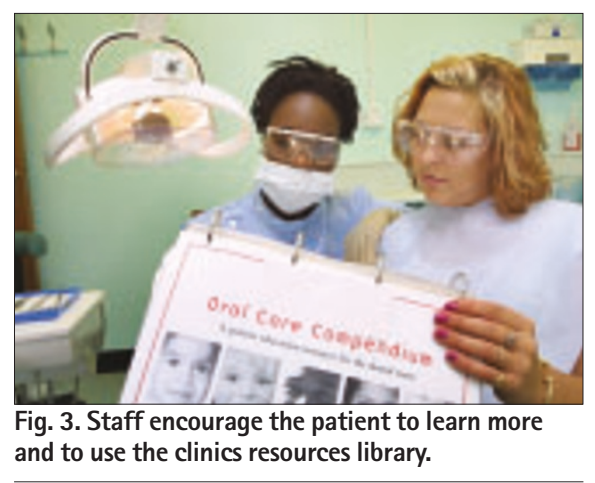

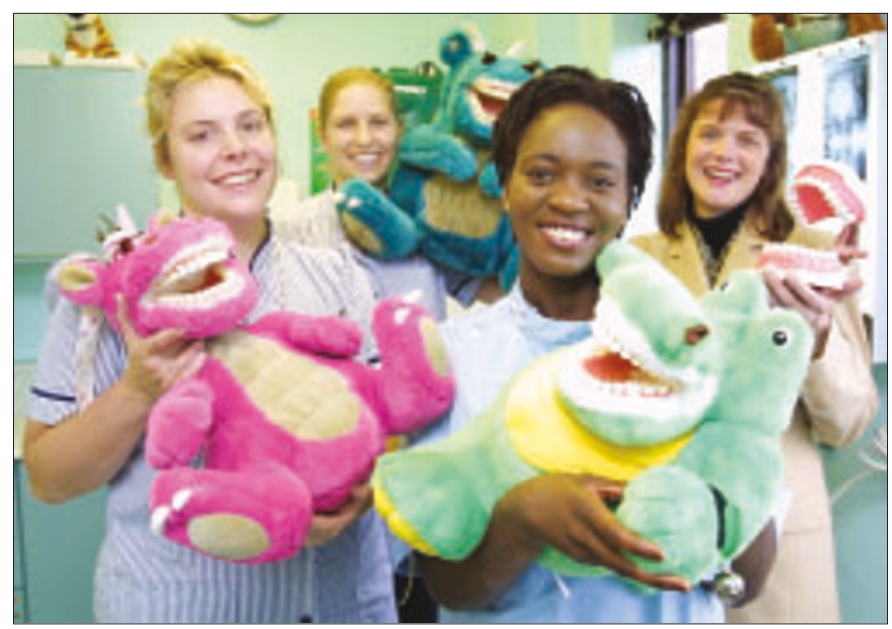

Fig. 1. The team from Strelley Health Centre in Nottingham making the presentation of the waiting area more user friendly. Visual, informative and interactive displays were erected, and are regularly updated, to raise awareness of the oral health of the community. Interactive activities were also introduced to encourage patients and carers alike to learn and be busy while in the reception area.

Visual aids and learning resources Visual aids were introduced in the clinics to help with specific clients. For example, the use of puppets, bubbles, musical instruments and other toys really catch the attention of special care patients, especially while they are being treated. The clinics have many such patients and even some phobics, and have found such aids to be very effective. The idea is that the patient and carer will be more relaxed and enable the dental professional to administer valuable treatment for the benefit of both the patient and carer.

A resource library was initiated to replenish a large gap of 'informative need' in the community. A selection of oral health resources is now accessed freely and at no cost by the community or the dental professionals. The CDS staff have promoted the resource library by distributing flyers throughout the community.

Delivering the oral health message Individual oral health training workshops were initiated throughout the Nottingham community dental service with the intention of training staff in their own and other clinics to deliver oral health messages effectively. Each workshop offers interactive oral health promotion and provides information and resources. The aim of these workshops is to raise positive awareness of issues such as preventative measures, oral hygiene, problems and treatments and healthy diets while adapting each workshop to suit the group's personal requirements.

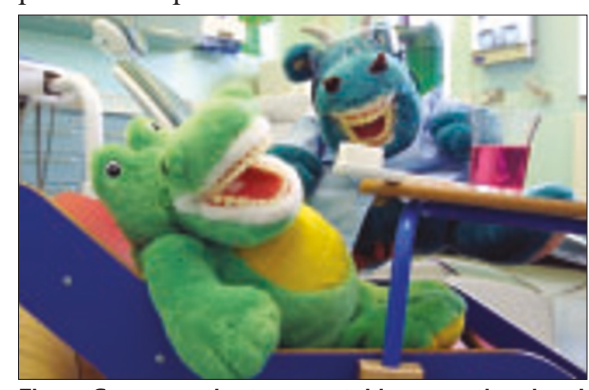

Fig. 4. Some say the toys are taking over the place! 